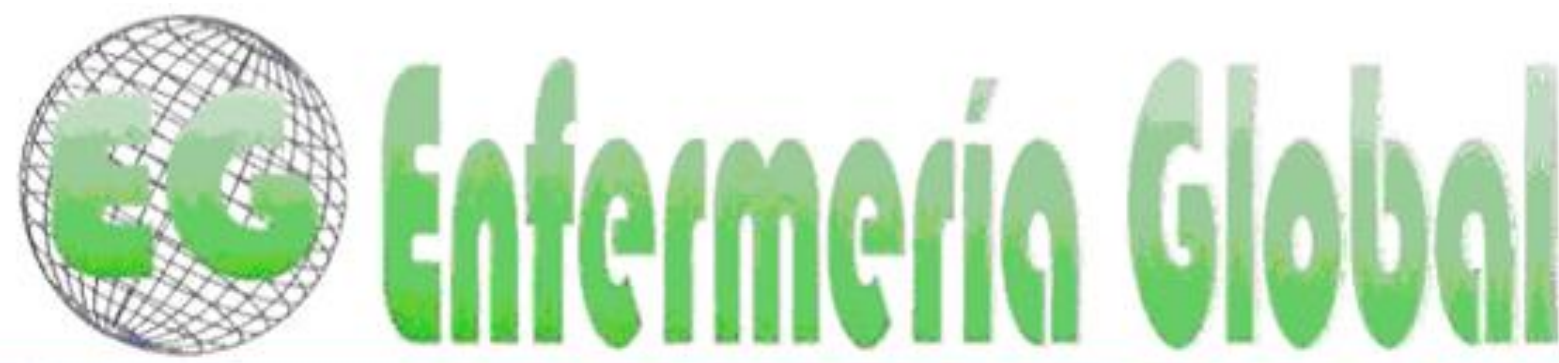

ISSN 1695-6141

Revista electrónica trimestral de Enfermeria

$N^{\circ} 34$

Abril 2014

www.um.es/egloball

ENSAYOS

\title{
Modelos de Enfermería en Unidades de Paciente Crítico: un paso hacia el cuidado avanzado
}

Nursing Models in Critical Care Units: Step toward advanced nursing care

\section{*Avilés Reinoso, Lissette Alejandra *Soto Núñez, Claudia}

\author{
*Enfermera. Tesista de Magíster en Enfermería. Pontificia Universidad Católica de Chile E-mail: \\ llaviles@uc.cl
}

Palabras clave: modelos de enfermería; unidades paciente crítico; cuidado avanzado.

Keywords: nursing models; critical care units; advanced nursing care.

\section{RESUMEN}

Este artículo tiene el objetivo de reflexionar sobre la utilización de modelos de enfermería en las unidades de paciente crítico en Chile, con el fin de brindar cuidados avanzados en estas unidades. Los cuidados avanzados de enfermería se sustentan en la utilización de modelos enfermería en la práctica clínica que permitan una entrega de cuidado de calidad, con sustento teórico de enfermería. Las unidades de paciente crítico son unidades que se relacionan con atención de enfermería centrada en la tarea, en el manejo de la tecnología y actividades rutinarias. En este escenario es necesario identificar modelos de enfermería que puedan implementarse en estas unidades y de esta forma ser un paso hacia el cuidado avanzado de enfermería en unidades de paciente crítico.

\section{ABSTRACT}

This article aims to reflect on the use of nursing models in critical patient units in Chile, in order to provide advanced care in these units. The advanced nursing care is based on the use of nursing models in clinical practice to enable delivery of quality care with nursing theoretical support. Critical patient units are units that are related to nursing care focused on the task, in the management of technology and routine activities. In this scenario it is necessary to identify nursing models that can be implemented in these units and thus be a step toward advanced nursing care in critical patient units.

\section{I.INTRODUCCIÓN}

La práctica avanzada de enfermería, es un concepto aún incipiente en Chile. Campos define los cuidados avanzados como aquellos destinados a mejorar cualitativamente la práctica autónoma de la profesión de enfermería, sustentados en conocimientos desarrollados por la disciplina ${ }^{(1)}$. Existen avances desde la academia para desarrollar 
el concepto, sin embargo, su utilización en la práctica es aún escasa. Uno de los elementos centrales en el cuidado avanzado es entonces, la utilización de modelos y/o teorías de enfermería en la práctica.

De esta manera, el uso de modelos de enfermería permite profundizar y crear nuevos conocimientos a partir de la experiencia personal en la clínica. Esto genera un lenguaje común en un paradigma compartido en enfermería que da paso a la reflexión, a la investigación, al crecimiento de la disciplina y además, mejora la satisfacción de los pacientes ${ }^{(2,3,4)}$. Lamentablemente, diversos estudios dan cuenta de que su aplicación no es reconocida como útil por los enfermeros clínicos ${ }^{(5,6)}$. No obstante, existen interesantes reportes en los Estados Unidos en el desarrollo de modelos de enfermería aplicados en estas unidades. Un ejemplo de ello es el modelo Primary Nursing, desarrollado por Marie Manthey en la década de los 80 y que se ha aplicado con éxito en las unidades de paciente crítico (UPC) ${ }^{(7,8)}$.

Las UPC han sido consideradas, tradicionalmente, como áreas estresantes y despersonalizadas debido a múltiples factores: pacientes con riesgo vital inminente que son separados abruptamente de su familia; dependencia de diversos elementos invasivos; ambiente altamente tecnologizado e incomprensible para todo el que ahí no trabaja. A partir de ello se dan características especiales del cuidado de enfermería que van a repercutir profundamente en el paciente, en su familia y en el mismo profesional $^{(9,10)}$.

La ausencia de modelos de enfermería adquiere mayor relevancia en las UPC, donde el cuidado de los pacientes es más complejo, debido a su dependencia y vulnerabilidad. Esto puede deberse a diferentes causas, entre ellas, la sobrecarga de trabajo, la sobrevaloración del modelo biomédico y el desconocimiento de los modelos de enfermería y su utilidad en la práctica ${ }^{(5,6,11)}$. Como consecuencia de ello, la atención de enfermería se centra en la tarea y en actividades derivadas del diagnostico médico, en el manejo de la tecnología, actividades rutinarias, parcializadas y mecanizadas. De esta manera, la necesidad de sustentar la práctica clínica de las UPC en fundamentos teóricos de la disciplina permitiría evitar que la práctica se centre en la tarea y genere un cuidado vacío.

En este contexto, uno de los problemas frecuentes es el desconocimiento de la utilidad de modelos de enfermería y su aplicación en la práctica, especialmente en UPC. Para ello, es necesario realizar intervenciones educativas cuyo objetivo final sea implementar modelos de enfermería que contribuyan al cuidado profesional y de esta forma ser un paso hacia el cuidado avanzado de enfermería en unidades de paciente crítico. Esto podría contribuir a mejorar la práctica de la disciplina en estas unidades y mejorar la satisfacción usuaria y de las enfermeras ${ }^{(12,13,14)}$.

\section{II.DESARROLLO}

Los cuidados avanzados de enfermería comenzaron a desarrollarse en la década de los 60 en Estados Unidos, con un fuerte desarrollo en Inglaterra, Australia y Canadá. En Latinoamérica no obstante, su evolución y desarrollo es incipiente. La definición de cuidados avanzados que identifica plenamente el propósito de este trabajo, es la declarada por diferentes autores, para los cuales, los cuidados de enfermería deben estar apoyados por un marco teórico propio de la disciplina, con el objetivo de entregar cuidados holísticos a los pacientes ${ }^{(1)}$. Al mencionar un "saber propio de la disciplina", se refieren a un modelo o teoría probada científicamente, diseñada por 
una enfermera en base a su conocimiento y experiencia. Lamentablemente una debilidad que poseen las enfermeras en su práctica clínica es el desconocimiento y la no utilización de teorías o modelos de enfermería que sustente su quehacer ${ }^{(5,6)}$. Existe la creencia de que la aplicación de estos modelos son complejos y demandan mucho tiempo especialmente al ser escritos ${ }^{(15)}$. Pero, la utilidad de los beneficios que entregan estos marcos de referencia en el desarrollo de la profesión, son variados y comprobados ya que permiten la creación de conceptos y propuestas generalizables y consensuadas para la atención del paciente, proporcionan hipótesis verificables, integran conocimientos de otras disciplinas, describen un fenómeno entre sí porque lo relacionan y orientan en la mejora de la práctica en cuanto son creados a partir de la observación ${ }^{(2,3,4)}$.

La ausencia de modelos de enfermería adquiere mayor relevancia en las unidades de paciente crítico, donde el cuidado de los pacientes es más complejo, debido a su dependencia y vulnerabilidad. Las UPC se han asociado a unidades en las cuales "la tecnología y la eficacia práctica predominan sobre las relaciones personales y la calidad humana" ${ }^{(4)}$. De esta manera, estas unidades altamente tecnologizadas se han relacionado con deshumanización y despersonalización por parte de los equipos de salud $(16,17,18)$. Algunos autores han planteado el desafío que supone para las enfermeras armonizar entre el paradigma científico y el paradigma humanista, es decir, armonizar la tecnología con el cuidado. El desafío se plantea entonces, en como la enfermera utiliza de forma adecuada la tecnología y la pone al servicio del cuidado de las personas, en especial en este tipo de escenarios ${ }^{(19)}$.

Las unidades de paciente crítico constituyen las unidades de mayor complejidad dentro de los centros hospitalarios de salud. Tienen cupos reducidos, a los cuales ingresan pacientes graves que necesitan asistencia en terapias específicas como ventilación mecánica, asistencia hemodinámica, cardiovascular, renal, entre otras. Poseen por ello alta especificidad tecnológica y de recursos, tanto materiales como de personal.

La distribución general del personal de enfermería, es de una enfermera por cada tres pacientes ${ }^{(20)}$, siendo parte de las funciones de las enfermeras el cuidado clínico, dentro de ello, la monitorización del paciente, cumplimiento del tratamiento médico, realización de procedimientos, planificación del cuidado, entre otras. En cuanto a actividades autónomas de la profesión se encuentra, principalmente, la aplicación del proceso de enfermería en cada uno de los pacientes a su cargo, de lo que se desprende el plan de enfermería dirigido al personal técnico, es decir, el control de signos vitales, programación de prevención de lesiones de la piel, confort del paciente, entre otras. Actividades orientadas a la gestión son la coordinación con otros profesionales y servicios de apoyo a la unidad y actividades administrativas como el suministro de insumos, medicamentos del día para los pacientes a cargo, etc.

Otra función fundamental de la enfermera en estas unidades es la evaluación constante del estado de los pacientes asignados a su cargo, con el fin de mantener su condición estable o pesquisar precozmente posibles complicaciones. Otra de las funciones importantes que desarrolla la enfermera en estas unidades es la interacción con la familia, ya sea dando información, explicación de los procedimientos y educación.

Por otro lado, el perfil de los pacientes que ingresan y permanecen en estas unidades debe ser considerado al elegir un modelo o teoría en particular, ya que presentan 
características particulares y que por tanto guían los cuidados brindados por los profesionales y en especial por las enfermeras. En cuanto a aspectos epidemiológicos de los pacientes de UPC, se observan altas tasas de mortalidad, de los que sobreviven al año fallecen entre 26 y $63 \%$; y el $33 \%$ de ellos, nunca vuelve a trabajar debido a la alta dependencia de las actividades diarias al alta. En cuanto a características psicológicas evaluadas posterior al alta, entre el 10 y $50 \%$ de los pacientes presentan síntomas de depresión, ansiedad, estrés post traumático y problemas de sueño. Y sus familiares directos, el $70 \%$ sufre síntomas de ansiedad y el tercio puede sufrir síntomas de depresión y estrés post traumático ${ }^{(13,21)}$.

Por otro lado, estudios han abordado la relación que existe entre los enfermeros y los familiares de pacientes en estas unidades, encontrando una comunicación insuficiente entre ambos. Sin embargo, estos mismos estudios describen el deseo de los enfermeros de participar más en la entrega de información a las familiares, como así también flexibilizar los horarios restrictivos en estas unidades ${ }^{(22)}$.

De esta manera, los modelos de enfermería son un medio necesario para el desarrollo de la disciplina y forman parte esencial para brindar un cuidado avanzado en enfermería. Para Lescaille ${ }^{(23)}$ la ética del cuidado se basa en respetar el sufrimiento del otro, ser sensible a su dolor, protegerlo a él y su familia y hacerle sentir lo menos posible su discapacidad o dependencia. De esta forma, el cuidar se configura como un acto inherentemente ético, puesto que involucra el respeto a la dignidad de la persona que requiere de dicho cuidado y el respeto a la dignidad misma del enfermero que lo otorga.

La ética del cuidado, entonces, se encuentra implícita en los modelos en cuanto propicia y guía un cuidado centrado en la persona como ser digno. Ofrece de esta manera un marco teórico y ético del cuidado que la enfermera en su condición profesional, moral y ética debe otorgar. Además de reforzar su condición social y valórica de servicio a la sociedad. El cuidar involucra necesariamente no dañar a otros, y esto se realiza a través de la responsabilidad autoescogida para brindarlo, en cuanto se elige esta profesión y se desea otorgar un cuidado profesional ${ }^{(24)}$.

Se han descrito en la literatura una gran teoría, una teoría de rango medio y un modelo de enfermería que han sido aplicados en unidades de paciente crítico.

El modelo de Virginia Henderson ha sido probado en la práctica clínica y podría ser de gran utilidad en las UPC, ya que está orientado principalmente al rol asistencial de la enfermera. Establece 14 componentes a evaluar en la atención de los pacientes, lo que permite una valoración integral de la persona tomando en cuenta su entorno y recuperación. Este puede ser aplicable a través del Proceso de Enfermería (PE), a través de la descripción de una relación enfermera-paciente como "sustituta" cuando el paciente es totalmente dependiente, establece un rol docente para enfermería y además considera la atención digna en el proceso de la muerte ${ }^{(25)}$. Este modelo es uno de los más reconocidos por las enfermeras clínicas, existiendo una serie de experiencias descritas, especialmente asociado al PE, a registros de enfermería y como modelo a seguir en escuelas de enfermería a nivel mundial y nacional ${ }^{(26,27,28,29)}$.

La teoría de mediano rango basada en el modelo de adaptación de Roy y la teoría organizacional de Weick, "Facilitar el hacer sentido en familiares de pacientes en UPC", de Judy Davidson ${ }^{(12)}$, es un modelo práctico y comprobado en familiares de pacientes de unidades de cuidados intensivos (UCI). A través de una buena 
comunicación, sencillas instrucciones y actividades orientadas al confort y recreación del paciente por parte de la enfermera, se pretende disminuir los efectos psicológicos adversos (depresión, stress y trauma post traumático) del familiar al sentirse útil y contribuir con el bienestar de su ser querido. Incluso, a partir de esta teoría, se establecieron guías de prácticas clínicas para el soporte de familiares de pacientes de $\mathrm{UCI}$, del Colegio de Medicina Intensiva en USA ${ }^{(30)}$.

Un modelo de enfermería que se ha utilizado en unidades con pacientes de estadía prolongada es el modelo Primary nursing o "enfermera de cabecera", creado por Marie Manthey a fines de la década de los 60. Este modelo propone cuidados de enfermería centrados en el paciente a través de una relación interpersonal y humana $^{(13)}$, mientras la enfermera se empodera de su rol. Existen cuatro principios fundamentales en este modelo: 1) Responsabilidad, donde una enfermera se hace responsable del cuidado integral de un grupo de pacientes de acuerdo a sus necesidades; 2) Método de caso, la enfermera es asignada a un número determinado de pacientes, de los cuales organiza y coordina todas los cuidados; 3) Comunicación, la enfermera es la interlocutora de los cuidados de sus pacientes con el resto del equipo de salud, con el paciente y su familia; 4) Continuidad en el cuidado, independiente de los turnos, es la enfermera de cabecera la que vela por la continuidad de los cuidados desde el ingreso del paciente al alta ${ }^{(31)}$. Este modelo ha sido ampliamente probado y documentado con buenos resultados en la práctica clínica, especialmente en pacientes crónicos ${ }^{(13,32,33)}$. En unidades de paciente crítico, Goode \& Rowe ${ }^{(8)}$ reporta su uso y la mejora en aspectos como la calidad y seguridad de la atención de los pacientes. Sin embargo, destaca el desgaste emocional que sufren las enfermeras producto del vínculo y el tiempo prolongado con pacientes graves, de los cuales muchos fallecen.

\section{CONCLUSIONES}

Implementar un modelo de enfermería en las unidades de paciente crítico para la práctica clínica diaria, si bien parece una utopía, puede ser una realidad, si las enfermeras se proponen incorporar conocimiento disciplinar a su práctica. El conocimiento de la práctica avanzada debe ser un paso inicial para generar el cambio y hacer conciencia de que un cuidado profesional y de calidad implica necesariamente la utilización de teorías y modelos de enfermería. Si bien no está exento de esfuerzo y dedicación, aspectos claves son contar con el apoyo institucional, el conocimiento de una docente o enfermera experta y un liderazgo persistente y motivador de manera de transmitirlo al equipo con el que desea trabajar.

La práctica basada en evidencia, a través de experiencias ya efectuadas y el compartir con enfermeras que ya han implementado el modelo deseado, es otra estrategia a seguir, especialmente para ver aspectos relacionados con la aplicación práctica.

El seguimiento, la evaluación y la retroalimentación podría permitir la utilización continua de modelos de cuidados, y si es necesario efectuar cambios, modificaciones y ajustes al mismo. El desarrollo de modelos en unidades de paciente crítico es una iniciativa que es posible de desarrollar como un proyecto innovador y pionero en el área de unidades de pacientes críticos en Chile, que incluya en el centro a sus pacientes y familias, y que permita un desarrollo disciplinar para los enfermeros en el país. 


\section{REFERENCIAS BIBLIOGRÁFICAS}

(1) Campos M. Cuidados de enfermería avanzados. Rev Horizonte enferm 2007; 18(1):51-6.

(2) López M. et al. Reflexiones acerca del uso y utilidad de los modelos y teorías de enfermería en la práctica asistencial. Enferm Clín 2006;16(4): 218-21.

(3) Mejías P. Aplicación de algunas teorías de enfermería en la práctica clínica. Index Enferm 2008; 17(3): 197-200.

(4) Pérez M. et al. Valoración del grado de satisfacción de los familiares de pacientes ingresados en una unidad de cuidados intensivos. Med Inten 2004; 28(5):237-49.

(5) Santos S. López M. Varez S. Abril D. Propuesta de un modelo teórico para la práctica enfermera. Nur Inves 2010; 7(44). Disponible en:

http://www.fuden.es/FICHEROS ADMINISTRADOR/ORIGINAL/promodenf.pdf

(6) Santos S. et al. Modelos teóricos y estudiantes de enfermería: aspectos motivacionales para su elección durante las prácticas clínicas. Educ Méd 2011;14(2): 119-27.

(7) Manley K. Hamil J. Hanlon M. Nursing staff's perceptions and experiences of primary nursing practice in intensive care 4 years on. J Clin Nurs 1997; 6(4):277-87.

(8) Goode D. Rowe K. Perceptions and experiences of primary nursing in an UCl: a combined methods approach. Intensive critical care nursing 2001.17(5), 294-303.

(9) Ramirez C. Parra M. Percepción de los comportamientos de cuidado de enfermería en la unidad de cuidados intensivos. Av Enferm 2011; 29(1):97-108.

(10) Talamares et al. Elaboración de un cuestionario para medir la calidad con los cuidados de enfermería en unidades de cuidados intensivos cardiológicos desde la percepción de los pacientes. Enfer Cardiol 2009; Año XVI(47-48): 71-9.

(11) Molina P. Jara P. El saber práctico en Enfermería. Rev Cub Enferm 2010;26(2) Disponible en: http://scielo.sld.cu/scielo.php?script=sci arttext\&pid=S08643192010000200005\&lng=es.

(12) Davidson J. Facilitated sensemaking a strategy and new middle- range theory support families of intensive care unit patients. Crit Car Nurs 2010; 30(6): 28-39.

(13) Palma E. Catoni I. Arechabala M. Brantes L. Primary nursing para el cuidado de usuarios en hemodiálisis crónica. Hisp Health Care Inter 2006;(4)4: 203-09.

(14) Rivero P. Hernández O. Sáenz A. Reguera A. Meneses T. Enfermería de práctica avanzada: historia y definición. Enferm Clín 2002;12(6): 286-89.

(15) Moreno M. Importancia de los modelos conceptuales y teorías de enfermería: experiencia de la Facultad de Enfermería de la Universidad de La Sabana. Rev Aquichan 2005; 5(1). Disponible en: http://www.scielo.org.co/scielo.php?pid=S1657$\underline{59972005000100005 \& s c r i p t=s c i \text { arttext\&tlng }=e s}$

(16) Beltrán O. La unidad de cuidado intensivo, un lugar para quedarse solo. Inv Educ Enferm 2009; 27 (1): 34-45.

(17) Solano M. Las vivencias de paciente coronario en la unidad de cuidados críticos. Index de Enfermería 2005; 14(51):29-33.

(18) Vargas R. Cuidado humanizado. Al paciente críticamente enfermo: Enfermería pieza clave en la atención. Ciencia y Cuidado 2007; 4(4):21-7.

(19) Barnard A. Sandelowski M. Technology and human nursing care: (ir) reconcilable or invented difference? J Advan Nurs 2001;34(3):367-75.

(20) Sáez E. et al. Guías 2004 para el funcionamiento de unidades de paciente crítico. Rev Chil Med Int 2004;19(4):209-23.

(21) Harvey M. Davidson J. Long- term consequences of critical illness: A news opportunity for high - impact critical care nurse. Crit Care Nurs 2011;31(5):12-5. 
(22) Zaforteza C. et al. Relación entre enfermeras de cuidados intensivos y familiares: Indicios para el cambio. Nure Invest 2004; 3. [Acceso 10 de octubre 2012] Disponible en: http://www.nureinvestigacion.es/ficheros administrador/original/original3.pdf (23) Lescaille M. Relación de la ética del cuidar y los modelos de enfermería con la persona y su dignidad. Rev Cub Enfer 2006;22(1):1-8. [Acceso 5 de Octubre 2012] Disponible en:

03192006000100008\&script=sci_arttext

(24) Santacruz M. Ética del cuidado. Universidad del Cauca. [Acceso 20 de Octubre 2012] Disponible en: http://facultadsalud.unicauca.edu.co/fcs/2006/junio/ETICA\%20Y\%20CUIDADO.pdf (25) Marriner A. Raile M. Modelos y teorías en enfermería. 6a ed. Barcelona; Elsevier: 2007.

(26) Vallejo J. Rodríguez M. Valverde M. Un modelo de registro en residencias de ancianos. Gerokomos 2007; 18 (2). [Acceso 12 de Octubre 2012] Disponible en: http://scielo.isciii.es/pdf/geroko/v18n2/72rincon.pdf

(27) Coll M. et al. Cuidados de Enfermería de acuerdo a V. Henderson en cuidados domiciliarios. Rev Rol Enfer 2007; 30(3):213-16.

(28) Estilleiro A. Ferrer M. Prevalencia de diagnósticos enfermeros de la NANDA y necesidades alteradas de Henderson en una unidad de lactantes. Rev Enfer Clín 2002;12(6): 253-59.

(29) Reyes J. Jara P. Merino M. Adherencia de las enfermeras/os a utilizar un modelo teórico como base de la valoración de enfermería. Rev Cienc Enfer 2007;13(1): 45-57. (30) Davidson J. et al. Clinical practice guidelines for support of the family in the patient-centered intensive care unit: American College of Critical Care Medicine Task Force 2004-2005. Crit Car Med 2007;35(2). [Acceso 20 de Septiembre 2012] Disponible

en: http://citeseerx.ist.psu.edu/viewdoc/download?doi=10.1.1.175.8195\&rep=rep1\&type=p df

(31) Manthey M. The practice of primary nursing. EEUU; Hallyday Lithograph Corporation: 1980

(32) Flett A. Introducción de enfermería primaria en un Centro satélite de diálisis en Singapur. EDTA-ERCA Journal 1997;23(2): 41-4.

(33) Laakso S. Routasalo P. Changing to primary nursing in a nursing home in Finland: Experiences of residents their family members and nurses. J Adv Nurs 2001; 33(4): 467-74.

ISSN 1695-6141

๑ COPYRIGHT Servicio de Publicaciones - Universidad de Murcia 